\title{
Adopción y difusión de la agricultura ecológica en España. Factores de reconversión en el olivar andaluz
}

\author{
Juan Agustín Franco Martínez \\ Escuela de Ingenierías Agrarias. Universidad de Extremadura \\ Carretera de Cáceres, $s / n$. \\ 06071 Badajoz \\ Tlf.: +34924289300 (ext. 86266). Fax: +34924286201 \\ franco@unex.es \\ Macario Rodríguez Entrena \\ Departamento de Economía y Sociología Agraria \\ Centro de Investigación Agraria CIFA-Granada \\ IFAPA - Junta de Andalucía \\ Camino Purchil, s/n \\ 18080 Granada \\ macario.rodriguez.ext@juntadeandalucia.es
}

\begin{abstract}
RESUMEN
Este artículo estudia las características del proceso de adopción y difusión de la agricultura ecológica en España, particularmente del olivar ecológico andaluz, para ello se revisa la legislación comunitaria y se realiza un análisis empírico de la adopción conjunta de medidas relacionadas con la producción ecológica y la lucha contra la erosión del suelo, partiendo de una base de datos que integra a más de doscientos agricultores de las provincias de Granada y Jaén en 2004. Concretamente, se realiza un análisis de los factores que determinan la decisión de reconvertir la explotación hacia un sistema de producción ecológica mediante la estimación de un modelo probit. También se estiman varios modelos sigmoidales de difusión de la práctica de conservación de suelos más implementada en la zona de estudio (mantenimiento de pedrizas o muretes). De los resultados obtenidos se concluye que es necesario reforzar más en la
\end{abstract}


legislación agroambiental la conexión entre adopción conjunta de prácticas de conservación de suelos complementarias y sistema de producción ecológica.

Palabras clave: agricultura ecológica, erosión del suelo, olivar andaluz, procesos de adopción y difusión, prácticas de conservación de suelos.

Clasificación JEL: Q12, Q16, Q24.

\begin{abstract}
This article studies of adoption and diffusion processes of Spanish ecological agriculture, mainly Andalusian ecological olive grove. For this, European agri-environmental law is revised and an empirical analysis about conjoint adoption of ecological production and fight against soil erosion is realized. Data carried out of a survey of two hundred farmers of Granada and Jaen in 2004. Specifically, factors that explain the decision of reconversion towards ecological production is studied by a probit model. Also several sigmoidal models of the principal soil conservation practice in the area of study (maintenance of stonewalls) is estimated. Finally to connect more tightly complementary soil conservation practices and ecological production is recommended.
\end{abstract}

Keywords: ecological agriculture, soil erosion, Andalusian olive groves, adoption and diffusion processes, soil conservation practices.

JEL Classification: Q12, Q16, Q24.

\title{
1. INTRODUCCIÓN
}

El sector ecológico en España se encuentra en fase de expansión desde la década de los noventa. En 2002 se superaron las seiscientas mil hectáreas certificadas y se contabilizaron casi dieciocho mil operadores. En 2005 con más del 15\% de la superficie ecológica comunitaria España ocupaba el segundo lugar en la Unión Europea, junto con Alemania y después de Italia (en este año las ventas de productos ecológicos en la UE ascendieron a 14 billones de euros). En 2008 se superó el millón de hectáreas bajo producción ecológica, alcanzándose también más de cien mil hectáreas de olivar ecológico, encontrándose entorno al 40\% de esta superficie olivarera en Andalucía desde el año 2001. En Andalucía es particularmente destacable el notable incremento de la superficie ecológica con respecto a la nacional, pasando del 22,14\% en 2001 al 59,50\% en 2008 (Tablas 1 y 2). 
ADOPCIÓN Y DIFUSIÓN DE LA AGRICULTURA ECOLÓGICA EN ESPAÑA.

FACTORES DE RECONVERSIÓN EN EL OLIVAR ANDALUZ

TABLA 1.-Datos básicos sobre producción ecológica en España (1991-2008)

\begin{tabular}{|c|c|c|c|c|c|c|}
\hline Año & Operadores & Productores & Elaboradores & $\begin{array}{c}\text { Superficie } \\
\text { ecológica } \\
\text { total (ha) } \\
\text { (1) }\end{array}$ & $\begin{array}{c}\text { Superficie } \\
\text { olivar } \\
\text { ecológico (ha) } \\
\text { (2) }\end{array}$ & $\begin{array}{c}\% \\
(2 / 1)\end{array}$ \\
\hline 1991 & 396 & 346 & 50 & 4.235 & 1.068 & 25,22 \\
\hline 1992 & 654 & 585 & 69 & 7.858 & 1.430 & 18,20 \\
\hline 1993 & 867 & 753 & 114 & 11.674 & 1.450 & 12,42 \\
\hline 1994 & 1.066 & 909 & 157 & 17.208 & 3.241 & 18,83 \\
\hline 1995 & 1.233 & 1.042 & 191 & 24.078 & 3.912 & 16,25 \\
\hline 1996 & 2.404 & 2.161 & 240 & 103.735 & 11.596 & 11,18 \\
\hline 1997 & 3.811 & 3.526 & 281 & 152.105 & 23.553 & 15,48 \\
\hline 1998 & 7.782 & 7.392 & 388 & 269.465 & 59.011 & 21,90 \\
\hline 1999 & 12.341 & 11.812 & 526 & 352.164 & 65.018 & 18,46 \\
\hline 2000 & 14.060 & 13.394 & 666 & 380.920 & 71.351 & 18,73 \\
\hline 2001 & 16.521 & 15.607 & 914 & 485.079 & 82.246 & 16,96 \\
\hline 2002 & 17.751 & 16.521 & 1.204 & 665.055 & 85.967 & 12,93 \\
\hline 2003 & 18.505 & 17.028 & 1.439 & 725.254 & 91.209 & 12,58 \\
\hline 2004 & 17.688 & 16.013 & 1.635 & 733.182 & 90.042 & 12,28 \\
\hline 2005 & 17.509 & 15.693 & 1.764 & 807.569 & 91.485 & 11,33 \\
\hline 2006 & 19.211 & 17.214 & 1.942 & 926.390 & 93.432 & 10,09 \\
\hline 2007 & 20.171 & 18.226 & 2.061 & 988.323 & 94.251 & 9,54 \\
\hline 2008 & 23.473 & 21.291 & 2.168 & 1.317 .752 & 101.268 & 7,68 \\
\hline
\end{tabular}

Fuente: Elaboración propia a partir de MAPA (varios años).

TABLA 2.-Datos básicos sobre producción ecológica en Andalucía (2001-2008)

\begin{tabular}{|c|c|c|c|c|c|c|c|c|}
\hline Año & Operadores & Productores & Elaboradores & $\begin{array}{c}\text { Superficie } \\
\text { ecológica } \\
\text { andaluza (ha) } \\
(3)\end{array}$ & $\begin{array}{c}\text { Superficie } \\
\text { olivar } \\
\text { ecológico } \\
\text { (ha) } \\
(4)\end{array}$ & $\begin{array}{c}\% \\
(3 / 1)\end{array}$ & $\begin{array}{c}\% \\
(4 / 3)\end{array}$ & $\begin{array}{c}\% \\
(4 / 2)\end{array}$ \\
\hline 2001 & 4.173 & 3.983 & 190 & 107.380 & 31.852 & 22,14 & 29,66 & 38,73 \\
\hline 2002 & 4.241 & 4.024 & 214 & 225.599 & 31.517 & 33,92 & 13,97 & 36,66 \\
\hline 2003 & 5.079 & 4.768 & 305 & 283.220 & 37.588 & 39,05 & 13,27 & 41,21 \\
\hline 2004 & 5.378 & 5.053 & 324 & 326.673 & 40.868 & 44,56 & 12,51 & 45,39 \\
\hline 2005 & 5.483 & 5.159 & 320 & 403.361 & 41.516 & 49,95 & 10,29 & 45,38 \\
\hline 2006 & 6.550 & 6.195 & 351 & 537.269 & 42.148 & 58,00 & 7,84 & 45,11 \\
\hline
\end{tabular}


TABLA 2.—Datos básicos sobre producción ecológica en Andalucía (2001-2008) (cont.)

\begin{tabular}{|l|l|l|l|l|l|l|l|l|}
\hline 2007 & 7.585 & 7.175 & 402 & 582.745 & 42.336 & 58,96 & 7,26 & 44,92 \\
\hline 2008 & 8.125 & 7.777 & 393 & 784.067 & 41.557 & 59,50 & 5,30 & 41,04 \\
\hline
\end{tabular}

Fuente: Elaboración propia a partir de MAPA (varios años).

La producción ecológica española es heterogénea y se dirige principalmente al mercado de exportación, aunque también van destacando gradualmente las asociaciones de consumidores y tiendas ecológicas de cara al mercado interior, cuyo desarrollo es más intenso en la CC.AA. catalana. Siendo Alemania, Francia y EEUU donde existen grandes cadenas de supermercados ecológicos. No obstante, la agricultura ecológica está adquiriendo cada vez mayor relevancia no sólo por su creciente dimensión económica, sino también ambiental y social. Así, la agricultura ecológica cumple una función básica de fijación de la población rural al territorio, principalmente en zonas marginales o deprimidas, donde la actividad agraria ya no se sustenta por sí sola a los precios del mercado internacional, debido, entre otras razones, a problemas de degradación de la tierra por causa de las malas prácticas agrarias.

Conviene recordar que el $40 \%$ del territorio español está afectado gravemente por la erosión, localizándose los mayores porcentajes de superficie erosionada en el suroeste peninsular (González y otros, 2005; Thorette y otros, 2005; Pimentel, 2006), siendo ya irrecuperable más de un 6\% (Rubio, 2006). En consecuencia, la agricultura ecológica se va imponiendo como una herramienta de gestión agroambiental y económica más adecuada, ya que se basa en un modelo productivo compatible con la conservación de los ecosistemas, el cual se ve reforzado por la adopción y difusión de buenas prácticas agrarias que frenan y previenen el problema de la erosión de suelos.

\section{ANTECEDENTES. LA DIFUSIÓN DE INNOVACIONES AGRARIAS}

La evolución histórica de las teorías del desarrollo agrícola se fundamenta en el supuesto básico de que el crecimiento agrícola es decisivo para la industrialización y el desarrollo económico de una nación. La Tabla 3 resume los modelos de innovación y adopción de tecnologías agrarias partiendo de los antecedentes del modelo clásico de Ryan y Gross (1943), para ir profundizando después en diversos aspectos desde una perspectiva histórica evolucionista, de manera que los sucesivos submodelos van aportando respuestas a problemas y cuestiones no resueltas por los anteriores. 
Siguiendo a Hayami y Ruttan (1971), pueden distinguirse tres modelos generales explicativos del desarrollo y la innovación agraria que caracterizan los antecedentes históricos del paradigma clásico de la difusión de innovaciones. Estos tres modelos son el de producción extensiva, el de producción intensiva y el de la industrialización.

El paradigma clásico de la difusión de innovaciones surge debido a las limitaciones de los modelos mencionados. Así, inicialmente se entiende la difusión de innovaciones (mejores prácticas agrícolas, mejores variedades de cultivos y de ganado, etc.) como la fuente más importante para el crecimiento de la productividad en la agricultura. Además, tiene gran importancia, no sólo la generación de innovaciones, sino su difusión. Existe amplia evidencia empírica sobre la relación que existe entre una divulgación y comunicación más eficaz del conocimiento técnico y una subsiguiente reducción de la dispersión de la productividad entre explotaciones y entre regiones agrícolas (Franco, 2009).

Los trabajos clásicos de Ryan y Gross (1943) y Griliches (1957 y 1960) estudian la difusión agregada de innovaciones basándose en patrones sigmoidales de difusión logística. 


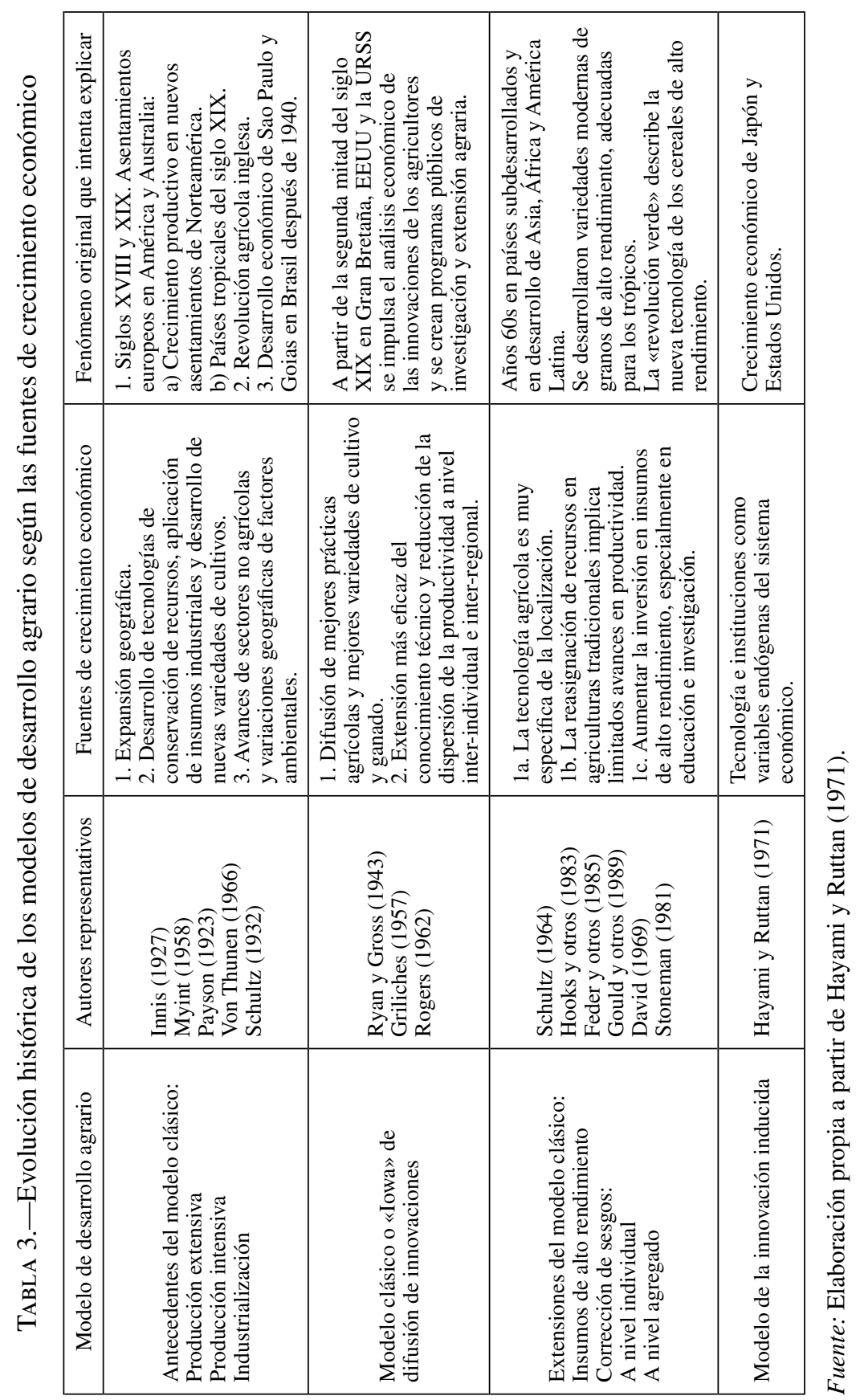


Aunque los modelos logísticos son los más habituales también se han aplicado otros modelos de difusión (Weibull, Gompertz, Richards, Morgan-Mercer-Flodin), incluyendo investigaciones basadas en la descomposición de la curva de crecimiento en varios componentes logísticos (Carrillo y González, 2002). La principal característica de los modelos logísticos es que se ajustan bien a las sendas de difusión cuya causa principal es el contacto entre los agentes del sistema en que se difunde (la información circula de unos agricultores a otros y la innovación se adopta por imitación). Griliches (1957) concluye que las variables económicas son los factores determinantes del patrón de cambio tecnológico, mientras que las variables sociológicas tienden a cancelarse entre sí en el largo plazo. Otras explicaciones sobre la forma sigmoidal del proceso de difusión de innovaciones son las que se atribuyen a diversas fuentes de heterogeneidad como el tamaño de la explotación, la cualificación del capital humano, el tipo de suelos, los rendimientos de los cultivos, la actitud hacia el riesgo, el modelo de gestión empresarial, etc.

Aunque hay trabajos recientes que analizan la adopción y difusión de la agricultura ecológica como innovación agraria (Gil y otros, 2000; Calatrava-Requena, 2002; Barea y Ruíz, 2002; Alonso y otros, 2002; Parra, 2003; Parra y Calatrava-Requena, 2005; Calatrava-Requena y González, 2008; Alonso y Simón, 2008), son menos numerosos los que estudian la adopción y difusión de innovaciones agrarias complementarias, como puede ser el caso de la producción ecológica y las técnicas de conservación de suelos, lo que contribuiría a un mejor diseño de políticas basadas en paquetes de medidas.

\section{Marco Legislativo}

La normativa básica comunitaria sobre el sistema de producción ecológica viene recogida en el Reglamento 834/2007 sobre producción y etiquetado de productos ecológicos, derogando el anterior Reglamento 2092/91 sobre la producción agrícola ecológica y su indicación en los productos agrarios y alimenticios. En ambos se expresa que el método de producción ecológica implica usos menos intensivos de la tierra, así como restricciones en la aplicación de fertilizantes, pesticidas, abonos y productos químicos de síntesis, y la adopción de prácticas de cultivo no erosivas.

Las tecnologías ecológicas se basan en la diversificación y la intensificación de las interacciones biológicas y los procesos naturales beneficiosos, de forma que activan el sistema biológico de nutrición de las plantas y la regulación de los organismos que potencialmente puedan convertirse en plagas. Por este motivo las normativas sobre producción ecológica intentan establecer apoyos que compensen los costes que pueda 
tener la puesta en marcha de estas nuevas tecnologías, así como compensar las posibles pérdidas económicas como consecuencia del tránsito del sistema de producción convencional al ecológico, ya que el proceso de conversión es un proceso prolongado en el tiempo en el que se establecen nuevas relaciones entre los diferentes componentes del agroecosistema, donde es frecuente la reducción de los niveles de productividad.

Con estas medidas la agricultura ecológica persigue la obtención de alimentos basados en sistemas productivos que compatibilicen la rentabilidad económica para el agricultor y el respeto al medioambiente. El creciente interés en el mercado por los productos ecológicos se debe en gran medida al aumento de la concienciación de los consumidores sobre la seguridad alimentaria y el desarrollo sostenible.

Posteriormente al Reglamento 2092/91, en 2004 la Comisión Europea publica el Plan de Acción Europeo para la Alimentación y la Agricultura Ecológica, en el que se recogen 21 iniciativas destinadas a incentivar y desarrollar el mercado ecológico mediante el aumento de su eficacia, transparencia y confianza de los consumidores. Este plan de acción es en el que se inspira el Reglamento 834/2007. Recientemente, la Comisión Europea ha lanzado una nueva campaña de sensibilización para promover la agricultura y producción ecológicas en Europa (Comisión Europea, 2008). A nivel nacional, el Plan Estratégico Estatal para la Agricultura Ecológica se hace eco del Plan de Acción Europeo, expresando la necesidad de integrar los mecanismos de apoyo a la agricultura ecológica dentro de los planes de desarrollo rural, estableciéndose una serie de compromisos básicos obligatorios para poder acogerse a las ayudas agroambientales relativas a la producción ecológica.

A nivel autonómico, y en el marco del PDR (2007-2013) de Andalucía, destaca el Plan Estratégico de la Agricultura Ecológica Andaluza (2007-2013), el cual es una continuación del primer Plan Andaluz de Agricultura Ecológica (2002-2006). Los sistemas de producción ecológicos se recogen en la Submedida 214 (agricultura ecológica: 214-03 y ganadería ecológica: 214-04) del actual PDR andaluz. En él se establece que los compromisos básicos de los sistemas de producción ecológicos se refuerzan con compromisos adicionales cuyo objetivo principal es la conservación del suelo y del agua, mediante el uso de sistemas sostenibles de manejo del suelo (cubiertas vegetales en cultivos leñosos y mantenimiento de rastrojos en cultivos herbáceos). Es decir, el PDR andaluz integra en una única medida tanto la producción ecológica como la lucha contra la erosión. En cambio, otras Comunidades Autónomas como Murcia o Valencia han contemplado en sus respectivos PDR los aspectos agroambientales sobre la erosión del suelo como una línea específica de ayuda.

Por ejemplo, en el caso del olivar la superficie mínima de cultivo para poder percibir la ayuda es de 1 hectárea. Las ayudas previstas van destinadas a un doble objetivo, 
por un lado, a incentivar la conversión de la explotación desde el sistema convencional al ecológico; y por otro, constituyen un pago por los servicios ambientales y sociales que generan los agricultores ecológicos. La cuantía de la ayuda para el olivar no situado en pendiente es de 270,3 euros por hectárea, mientras que para el olivar en pendiente asciende a 370,4 euros por hectárea. El importe de la prima para las explotaciones que inicien el período de conversión será del $100 \%$ durante los tres primeros años de compromiso, mientras que para el cuarto y quinto año se reduce al 90\%. El presupuesto total previsto entre 2007 y 2013 que la Comunidad Autónoma de Andalucía destina para financiar las ayudas a la producción ecológica asciende a 92,5 millones de euros.

La Tabla 4 muestra la cuantía nominal y real de las primas concedidas por las diferentes normativas a la lucha contra la erosión en el olivar. Destaca la disminución, en términos constantes, de la prima a la Medida 4 del Reglamento 1257/99 con respecto al anterior. En cambio en el año 2008 el PDR (2007-2013) de Andalucía mejora en 20 euros la prima concedida al olivar no situado en pendiente después de los tres primeros años con respecto a la prima básica en 1993, mientras que supera los 60 euros/ha en el caso del olivar situado en pendiente.

TABLA 4.-Cuantía nominal y real (en euros por hectárea) de las primas concedidas a la lucha contra la erosión en el olivar

\begin{tabular}{|c|c|c|c|c|c|c|}
\hline Normativa & Tipo & rima & Cuantía nominal & Cuantía real & $\begin{array}{c}\text { Deflactor } \\
\text { PIBpm } \\
\text { (Base 1990) }\end{array}$ & Año \\
\hline \multirow{2}{*}{ R 2078/92 } & \multicolumn{2}{|c|}{ Básica } & 120,20 & 100,79 & \multirow{2}{*}{119,26} & \multirow{2}{*}{199} \\
\hline & \multicolumn{2}{|c|}{ Complementaria $(+30 \%)$} & 156,26 & 131,03 & & \\
\hline R $1257 / 99$ & \multicolumn{2}{|c|}{ Básica } & 132,22 & 88,24 & 149,85 & 2000 \\
\hline \multirow{4}{*}{$\begin{array}{c}\text { PDR } \\
\text { andaluz } \\
(2007-2013)\end{array}$} & \multirow{2}{*}{$\begin{array}{c}\text { Olivar no } \\
\text { situado en pte }\end{array}$} & Hasta $3^{\circ}$ año & 270,30 & 133,04 & \multirow{4}{*}{203,17} & \multirow{4}{*}{2008} \\
\hline & & $\begin{array}{c}4^{\circ} \text { y } 5^{\circ} \text { año } \\
(-10 \%)\end{array}$ & 243,27 & 119,74 & & \\
\hline & \multirow{2}{*}{$\begin{array}{l}\text { Olivar en } \\
\text { pendiente }\end{array}$} & Hasta $3^{\circ}$ año & 370,40 & 182,31 & & \\
\hline & & $\begin{array}{c}4^{\circ} \text { y } 5^{\circ} \text { año } \\
(-10 \%)\end{array}$ & 333,36 & 164,08 & & \\
\hline
\end{tabular}

Fuente: Elaboración propia.

Los compromisos generales que debe cumplir el agricultor que solicite las ayudas de la Submedida 214 se refieren al cumplimiento de las normas de producción establecidas en el Reglamento 2092/91 (aún vigente cuando se redactó el actual PDR andaluz), la elaboración de un plan de conversión de la explotación, mantener un cuaderno 
contable de la explotación, la realización de una actividad formativa sobre producción ecológica, mantener la explotación bajo el control de un organismo de certificación ecológica autorizado y comercializar la producción ecológica con el etiquetado correspondiente al finalizar la fase de reconversión.

Mientras que los compromisos específicos de la Submedida 214-03 de agricultura ecológica son los siguientes: No utilizar productos químicos de síntesis y controlar la maleza mediante tratamientos mecánicos. Emplear abono orgánico para mantener la fertilidad de los cultivos. Mantener la vegetación en las lindes de las parcelas. Mantener cubierta vegetal en el suelo de las calles entre el 1 de septiembre y el 15 de marzo. Para las labores de recolección se podrá desyerbar la superficie bajo la copa del árbol de forma mecánica entre el 1 de septiembre y el 15 de marzo. Y se prohíbe el uso de aperos de labranza que volteen el suelo y las roturaciones.

\section{Metodología}

En este epígrafe se expone la metodología seguida para el análisis de los procesos de adopción y difusión en el olivar ecológico andaluz, ya que, según ya se ha comentado, el mayor peso del sector ecológico se concentra en Andalucía y en el cultivo del olivar (donde, a su vez, también se localizan los mayores problemas de erosión). Es por lo que el estudio del olivar ecológico andaluz representa un indicador del desarrollo global del sector en España, así como de la expansión y difusión de prácticas de conservación de suelos.

Los datos utilizados en el análisis provienen de una encuesta realizada en 2004 a 223 olivicultores de diferentes municipios de las provincias de Granada y Jaén. El cuestionario constaba de varios bloques de preguntas relacionadas con las características de la explotación (superficie, rendimiento, pendiente, tipo de propiedad, manejo de ganado, sistema de cultivo, etc.), percepción de la erosión por el agricultor, prácticas de conservación, gestión y organización, participación en programas de la PAC y variables sociodemográficas (edad, nivel educativo, formación agraria, actitudes frente a la innovación, etc.).

En particular, se estima un modelo probit para determinar los factores que explican la decisión de los agricultores de reconvertir su explotación hacia olivar ecológico. Previo a la estimación de este modelo se realizó un test Chi-cuadrado para identificar las variables que estaban relacionadas significativamente con la decisión de reconvertir la explotación. Las variables no relacionadas fueron descartadas y no incluidas en el modelo. 
También se estiman diferentes modelos sigmoidales de difusión de la práctica de conservación de suelos relacionada con la decisión de reconversión hacia olivar ecológico (mantenimiento de pedrizas o muretes). El análisis de difusión de esta práctica se ha realizado estimando dos tipos de modelos sigmoidales de influencia interna (logístico y Gompertz) según dos enfoques: según agricultores (difusión inter-empresas) y según superficie (difusión global). La difusión inter-empresas representa el valor acumulado de nuevos adoptantes de una determinada práctica en cada período de tiempo respecto al total de agricultores de la muestra. La difusión global mide los incrementos sucesivos de superficie en la que anualmente se adopta la práctica.

Por último se realiza un ejercicio de simulación del proceso de difusión global de la superficie ecológica española total y olivarera, asumiendo un «escenario base» (relativamente optimista) que se justifica a partir de los datos comentados en la introducción de este artículo sobre crecimiento del sector y niveles de erosión. En el «escenario base» se ha supuesto una proporción en torno al $10 \%$ de la superficie ecológica olivarera sobre la superficie ecológica total para el período simulado y un techo máximo de crecimiento en ambas superficies (total y olivarera) del $80 \%$ de la superficie convencional.

\section{Resultados}

Los 223 agricultores encuestados engloban un total de 2.411,44 hectáreas de olivar. El 22,42\% de las explotaciones son de regadío. El tamaño medio de las explotaciones es de 10,81 hectáreas. La gran mayoría de las explotaciones se encuentran en régimen de propiedad (96\%). Y casi el $98 \%$ no realiza manejo de ganado en su explotación. La pendiente media de la finca es mayor del $15 \%$ para el $26,46 \%$ de las explotaciones encuestadas, entre el $8 \%$ y el $15 \%$ para el $52,47 \%$, e inferior al $8 \%$ para el $21,08 \%$.

Las principales prácticas adoptadas en la zona de estudio son el mínimo laboreo ( $28 \%$ de los agricultores y $25 \%$ de la superficie), el no laboreo con aplicación de herbicidas (59\% de los agricultores y $46 \%$ de la superficie) y el mantenimiento de pedrizas o muretes (19\% de los agricultores y de la superficie). Esta última se aplica en algunos casos de forma complementaria a una de las dos primeras. Y un $10 \%$ de los agricultores ( $25 \%$ de la superficie) declara realizar otras prácticas de conservación del suelo, como mantener taludes, vegetación natural o setos en las lindes, cubiertas vegetales o restos de poda triturados en las terrazas (Tabla 5). 
TABLA 5.-Distribución porcentual de las prácticas adoptadas en la zona de estudio (\% de agricultores y de superficie)

\begin{tabular}{|l|c|c|}
\hline \multicolumn{1}{|c|}{ Prácticas adoptadas } & \% Agricultores & \% Hectáreas \\
\hline Otras & 9,87 & 24,82 \\
\hline Sólo mínimo laboreo & 23,32 & 20,36 \\
\hline Mínimo laboreo y pedriza & 4,48 & 5,01 \\
\hline Sólo pedriza & 3,59 & 3,52 \\
\hline No laboreo y pedriza & 10,76 & 10,24 \\
\hline Sólo no laboreo & 47,98 & 36,05 \\
\hline
\end{tabular}

Casi la totalidad de los encuestados (98\%) considera que la rentabilidad de su olivar sin el sistema de ayuda a la producción es baja o muy baja. Por otro lado, el 63,35\% conoce las ayudas para la lucha contra la erosión, pero sólo participan en él aproximadamente el $40 \%$. Las razones argüidas para no participar fueron fundamentalmente dos: la complejidad de las prácticas exigidas $(48,10 \%)$ y la insuficiencia de los pagos $(21,52 \%)$.

Un tercio declara no conocer la agencia de extensión agraria de su comarca, y de los dos tercios restantes sólo el 37\% la visita, mayoritariamente para resolver trámites burocráticos relacionados con las ayudas de la UE. Es más, también se observa que los agricultores prefieren acudir a la cooperativa para resolver los problemas de plagas y enfermedades en su explotación (30,94\% de los encuestados) frente a los que deciden ir a consultar a la Oficina Comarcal Agraria o a un Centro de Investigación y Formación Agraria (3,14\%). Por último, con respecto al método de abonado el $60 \%$ lo realiza guiado por su propia experiencia o por la de agricultores vecinos, frente al $23 \%$ que prefiere aconsejarse a través de casas comerciales o acudiendo a la agencia de extensión agraria.

\subsection{El modelo probit de reconversión hacia olivar ecológico}

Un 23,77\% de los agricultores declara que en la próxima campaña va a iniciar la reconversión de su explotación hacia olivar ecológico. La Tabla 6 muestra la variable explicada y las explicativas utilizadas en el modelo de elección discreta estimado para determinar los factores que explican la decisión individual de reconvertir la finca hacia un sistema de producción ecológica. 
TABLA 6.-Descripción de las variables utilizadas en el modelo probit

\begin{tabular}{|l|l|c|c|}
\hline \multicolumn{1}{|c|}{ Variable } & \multicolumn{1}{|c|}{ Definición } & Proporción* & $\begin{array}{c}\text { Desviación } \\
\text { típica }\end{array}$ \\
\hline ECOLOG & (Variable dependiente): Reconversión a olivar ecológico (1/0) & 0,2377 & 0,4266 \\
\hline HAT $*$ & Superficie de la explotación (hectáreas) & 10,8136 & 18,9396 \\
\hline KGHA $*$ & Rendimiento de la explotación (kg/ha) & $3.806,2721$ & $2.508,2783$ \\
\hline PTE * & Pendiente de la explotación. 1: Alta; 2: Media; 3: Baja & 1,9635 & 0,6333 \\
\hline EROEXPL & Percepción grave de la erosión en su explotación (1/0) & 0,6188 & 0,4867 \\
\hline PEDR & Mantenimiento de pedrizas o muretes (1/0) & 0,1883 & 0,3918 \\
\hline NL & No laboreo con aplicación de herbicidas (1/0) & 0,5874 & 0,4934 \\
\hline PARTIC & Participa en el programa agroambiental (1/0) & 0,2421 & 0,4293 \\
\hline MAQPR & Maquinaria propia (1/0) & 0,4663 & 0,4999 \\
\hline CONT & Lleva la contabilidad él mismo (1/0) & 0,6278 & 0,4844 \\
\hline PRET & Lleva contabilidad sólo con fines impositivos (1/0) & 0,5695 & 0,4962 \\
\hline DESM & Dedicación a la actividad agraria secundaria/marginal (1/0) & 0,6143 & 0,4878 \\
\hline TRAB & Realiza todos los trabajos físicos en su explotación (1/0) & 0,7354 & 0,4420 \\
\hline SINFORM & Sin formación académica (1/0) & 0,4394 & 0,4974 \\
\hline
\end{tabular}

Nota: * En las variables cuantitativas se ha calculado la media aritmética.

Los resultados del modelo probit estimado para identificar los factores que explican la decisión del agricultor de reconvertir su explotación hacia olivar ecológico se muestran en las Tablas 7 y 8 , donde se recogen los coeficientes estimados, las proporciones de clasificación correcta, los efectos marginales y elasticidades. En el modelo estimado la prueba de la razón de verosimilitud indica que es significativo. Además, un alto porcentaje de los casos analizados fueron correctamente clasificados. El modelo presenta un buen valor del pseudo- $\mathrm{R}^{2}$ de $\mathrm{McFadden}$, todo lo cual indica un buen nivel de ajuste $\mathrm{y}$ un alto poder discriminante del mismo.

La Tabla 7 muestra que la probabilidad de reconvertir la finca hacia olivar ecológico es mayor cuando más grande es la superficie de la explotación, cuando el agricultor adopta la práctica de mantenimiento de pedrizas o muretes y cuando su dedicación a la agricultura es secundaria o marginal. Mientras que dicha probabilidad disminuye cuando mayores son los rendimientos de la explotación, cuando menor es la pendiente de la explotación, cuando el agricultor declara llevar él mismo la contabilidad de la explotación y lo hace sólo con fines meramente impositivos (cumplir las obligaciones con Hacienda). 
Otras variables como el nivel formativo, la participación en el programa agroambiental o la adopción de otras prácticas de conservación como el no laboreo no resultaron significativas.

TABLA 7.-Modelo probit binomial de «reconversión hacia olivar ecológico» (ECOLOG)

\begin{tabular}{|l|c|c|c|c|}
\hline \multicolumn{2}{|c}{ Variable } & \multicolumn{1}{c}{ Coeficiente } & \multicolumn{1}{c}{ p-valor } & \multicolumn{1}{c|}{ Efectos } \\
marginales & p-valor \\
\hline CONSTANTE & 1,1401 & 0,1953 & 0,2530 & 0,2017 \\
\hline HAT $*$ & 0,0147 & 0,0282 & 0,0032 & 0,0269 \\
\hline KGHA * & $-0,0001$ & 0,0579 & $-0,0002$ & 0,0596 \\
\hline PTE $*$ & $-1,0352$ & 0,0000 & $-0,2297$ & 0,0000 \\
\hline EROEXPL & $-0,1601$ & 0,6129 & $-0,0362$ & 0,6223 \\
\hline PEDR & 1,9772 & 0,0000 & 0,6263 & 0,0000 \\
\hline NL & 0,1725 & 0,5639 & 0,0376 & 0,5604 \\
\hline PARTIC & 0,2098 & 0,5152 & 0,0493 & 0,5349 \\
\hline MAQPR & $-0,2453$ & 0,3828 & $-0,0539$ & 0,3727 \\
\hline CONT & $-0,6849$ & 0,0145 & $-0,1664$ & 0,0201 \\
\hline PRET & $-0,8084$ & 0,0122 & $-0,1905$ & 0,0159 \\
\hline DESM & 0,9655 & 0,0034 & 0,1917 & 0,0009 \\
\hline TRAB & 0,1444 & 0,6615 & 0,0308 & 0,6483 \\
\hline SINFORM & $-0,2243$ & 0,3912 & $-0,0490$ & 0,3871 \\
\hline Razón de verosimilitud & 110,2302 & 0,0000 & & \\
\hline Pseudo-R ${ }^{2}$ de McFadden & 0,4507 & & & \\
\hline Predicciones correctas $(\%)$ & 89,238 & & & \\
\hline
\end{tabular}

Nota: Las elasticidades de las variables cuantitativas HAT, KGHA y PTE son respectivamente:

$$
\mathrm{E}_{\mathrm{HAT}, \mathrm{ECOLOG}}=0,2544 . \mathrm{E}_{\mathrm{KGHA}, \mathrm{ECOLOG}}=-0,5918 . \mathrm{E}_{\mathrm{PTE}, \mathrm{ECOLOG}}=-3,2362
$$

Los resultados del modelo comentado son coherentes con los obtenidos por Parra y Calatrava-Requena (2005) relativos a la orientación productiva (ecológica o no) de los olivicultores de la provincia de Córdoba, destacando la significatividad y la misma influencia de las variables de rendimientos y tiempo de dedicación a la agricultura.

Todas las variables significativas en el modelo estimado presentan igualmente efectos marginales significativos, correspondiendo los mayores efectos marginales a la pendiente de la explotación y a la adopción de pedrizas o muretes. Los valores de 
la elasticidad correspondientes a las variables continuas indican que la decisión de reconvertir la finca hacia olivar ecológico es inelástica ante cambios porcentuales en las variables de superficie olivarera y rendimientos, mientras que la respuesta es elástica con respecto a la pendiente.

TABLA 8.-Proporción de clasificación correcta del modelo probit ECOLOG

\begin{tabular}{|l|l|l|l|}
\hline & \multicolumn{2}{|l|}{ Predicción } & \\
\hline Observado & $\mathrm{Y}=0$ & $\mathrm{Y}=1$ & Total \\
\hline $\mathrm{Y}=0$ & 162 & 8 & 170 \\
\hline $\mathrm{Y}=1$ & 16 & 37 & 53 \\
\hline Total & 178 & 45 & 223 \\
\hline
\end{tabular}

Nota: Las predicciones del modelo se basan en un umbral $\mathrm{c}=0,5$

\subsection{La difusión de la práctica de mantenimiento de pedrizas o muretes}

Los modelos sigmoidales de influencia interna explican bien el proceso de difusión a través de interacciones entre los agricultores de la zona (efecto imitación), por esta razón se ha estimado una curva de crecimiento acumulado logística y otra Gompertz correspondientes a la difusión observada en la técnica de mantenimiento de pedrizas o muretes, que es la práctica conservacionista que ha mostrado una influencia significativa frente a la decisión de reconversión hacia olivar ecológico. Con propósitos comparativos también se ha estimado un modelo de influencia externa (modelo exponencial) y otro mixto (modelo de Bass). Los modelos no lineales no restringidos se han estimado mediante el método de Levenberg-Marquardt y los modelos restringidos mediante el método de programación cuadrática secuencial.

Las Figuras 1 y 2 muestran la estimación del proceso de difusión a nivel de agricultores y de superficie de la técnica de mantenimiento de pedrizas o muretes según diferentes curvas sigmoidales en comparación con los datos observados. El proceso de difusión de esta práctica se inició a mediados de los cuarenta, acelerándose el crecimiento a partir de la década de los sesenta, involucrando en 2003 aproximadamente al $20 \%$ de los agricultores y de la superficie de la zona de estudio, no habiéndose alcanzado aún el punto de inflexión a nivel de agricultores en el momento de realización de la encuesta. 
Figura 1.-Modelos de difusión a nivel de agricultores de la práctica de mantenimiento de pedrizas o muretes (1944-2003)

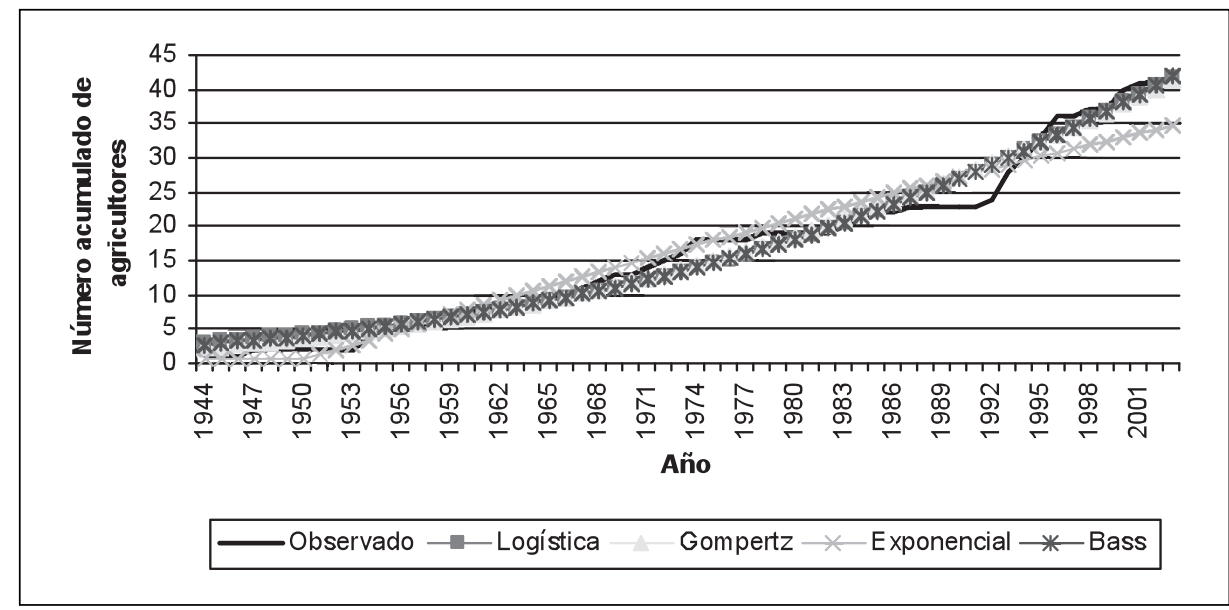

FIGURA 2.-Modelos de difusión a nivel de superficie de la práctica de mantenimiento de pedrizas o muretes (1944-2003)

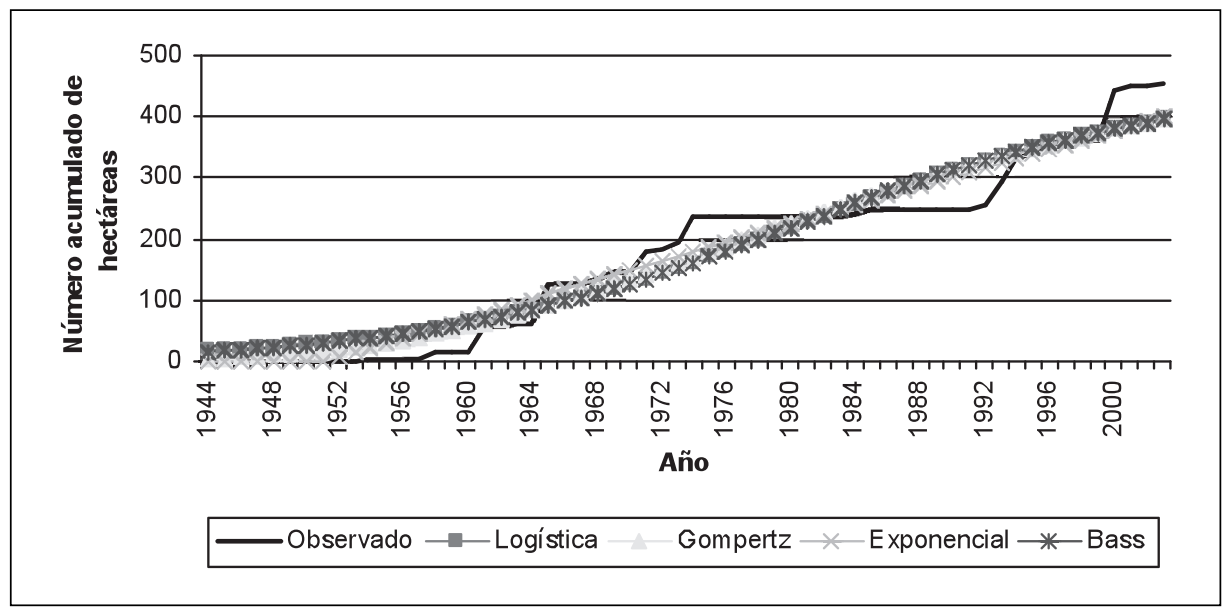

La Tabla 9 recoge los coeficientes de los parámetros estimados (siendo «M» el techo teórico que alcanzaría la práctica, «a» la constante de integración y «b» la tasa de difusión máxima), así como su error típico y el grado de ajuste del modelo. Se observa cómo los modelos de influencia interna (logístico y Gompertz) presentan mejores ajustes, siendo 
ligeramente inferiores a nivel de superficie. Las tasas máximas de difusión se corresponden con el modelo logístico o Verhulst-Pearl, lo que implica mayores velocidades de difusión debidas al efecto contagio, en contraste con las reducidas tasas exponenciales.

TABLA 9.-Modelos estimados de la práctica de mantenimiento de pedrizas o muretes

\begin{tabular}{|c|c|c|c|c|}
\hline \multirow{2}{*}{ Parámetros } & \multicolumn{4}{|c|}{ Modelo logístico: $\mathrm{M} /(1+\exp (\mathrm{a}-\mathrm{b} * \mathrm{t}))$} \\
\hline & Agricultores & Error típico & Superficie & Error típico \\
\hline $\mathrm{M}$ & 92,934 & 25,416 & 453,000 & 42,000 \\
\hline $\mathrm{a}$ & 3,383 & 0,200 & 3,294 & 0,249 \\
\hline $\mathrm{b}$ & 0,053 & 0,005 & 0,087 & 0,011 \\
\hline $\mathrm{R}^{2}$ & \multicolumn{2}{|c|}{0,971} & \multicolumn{2}{|c|}{0,924} \\
\hline \multirow{2}{*}{ Parámetros } & \multicolumn{4}{|c|}{ Modelo Gompertz: $\mathrm{M}^{*} \exp (-\exp (\mathrm{a}-\mathrm{b} * \mathrm{t}))$} \\
\hline & Agricultores & Error típico & Superficie & Error típico \\
\hline M & 223,000 & 117,963 & 520,282 & 64,887 \\
\hline $\mathrm{a}$ & 1,532 & 0,084 & 1,628 & 0,141 \\
\hline $\mathrm{b}$ & 0,017 & 0,004 & 0,049 & 0,008 \\
\hline $\mathrm{R}^{2}$ & \multicolumn{2}{|c|}{0,974} & \multicolumn{2}{|c|}{0,940} \\
\hline \multirow{2}{*}{ Parámetros } & \multicolumn{4}{|c|}{ Modelo exponencial: $\mathrm{M}-\exp (\mathrm{a}-\mathrm{b} * \mathrm{t})$} \\
\hline & Agricultores & Error típico & Superficie & Error típico \\
\hline M & 140,119 & 107,293 & $\underline{11.414,022}$ & $68.681,010$ \\
\hline $\mathrm{a}$ & 4,975 & 0,735 & 9,348 & 6,027 \\
\hline $\mathrm{b}$ & 0,005 & 0,005 & 0,001 & 0,004 \\
\hline $\mathrm{R}^{2}$ & \multicolumn{2}{|c|}{0,934} & \multicolumn{2}{|c|}{0,941} \\
\hline \multirow{2}{*}{ Parámetros } & \multicolumn{4}{|c|}{ Modelo de Bass: $\left(\mathrm{M}-\exp \left(\mathrm{q}-\mathrm{p}^{*} \mathrm{t}\right)\right) /\left(1+\exp \left(\mathrm{q}-\mathrm{p}^{*} \mathrm{t}\right)\right)$} \\
\hline & Agricultores & Error típico & Superficie & Error típico \\
\hline M & 105,433 & 33,380 & 453,000 & 41,847 \\
\hline $\begin{array}{c}\mathrm{q} \text { (influencia } \\
\text { interna) }\end{array}$ & 3,324 & 0,250 & 3,277 & 0,246 \\
\hline $\begin{array}{c}\mathrm{p} \text { (influencia } \\
\text { externa) }\end{array}$ & 0,049 & 0,004 & 0,087 & 0,011 \\
\hline $\mathrm{R}^{2}$ & \multicolumn{2}{|c|}{0,973} & \multicolumn{2}{|c|}{0,925} \\
\hline
\end{tabular}

Notas: Estimaciones realizadas con SPSS v.14. Parámetros significativos al 95\% de confianza. Restricción impuesta en los modelos Gompertz y exponencial a nivel de agricultores: $\mathrm{M}<=223$. Restricción impuesta en los modelos logístico y Bass a nivel de superficie: $\mathrm{M}>=453$. El modelo exponencial a nivel de superficie no es realista debido a que supera el techo máximo de la muestra analizada, establecido en 2.411,44 hectáreas. 
El modelo de Bass confirma lo comentado en los modelos anteriores sobre las tasas de difusión. Particularmente, los coeficientes de imitación son superiores a nivel de superficie que a nivel de agricultores, lo que implica que las explotaciones de mayor tamaño adoptan antes esta práctica.

La Tabla 10 resume los coeficientes de penetración en el mercado del mantenimiento de pedrizas. Anualmente por cada 17 adoptantes hay un imitador, mientras que en términos de superficie, por cada 50 hectáreas adoptadas, una nueva hectárea se convierte en adoptante de esta práctica.

Tabla 10.-Coeficientes de penetración (b/M) de la difusión logística de la práctica de mantenimiento de pedrizas o muretes en la zona de estudio

\begin{tabular}{|l|l|l|}
\hline & Agricultores & Superficie \\
\hline por cada $1 \%$ & $0,13 \%$ & $0,46 \%$ \\
\hline por cada unidad & $0,057 \%$ & $0,019 \%$ \\
\hline $\mathbf{M}^{*}$ & 41,675 & 18,785 \\
\hline $\mathrm{b}$ & 0,053 & 0,087 \\
\hline
\end{tabular}

Nota: * M calculado en porcentaje sobre el total muestral (223 encuestados y 2.411,44 has).

\subsection{La simulación de la difusión de la superficie ecológica}

Para realizar la simulación se perfilado un «escenario base» caracterizado por los siguientes supuestos: proporción en torno al $10 \%$ de la superficie ecológica olivarera sobre la superficie ecológica total para el período simulado y un techo máximo de crecimiento en ambas superficies (total y olivarera) del 80\% de la superficie convencional, suponiendo a partir de 2008 una banda de crecimiento estacionario entorno a los 25 millones de hectáreas totales de SAU y de 2,5 millones en olivar. 
Figura 3.-Estimaciones logísticas de la senda de difusión de la superficie ecológica total y olivarera en España en el escenario base (1991-2038)

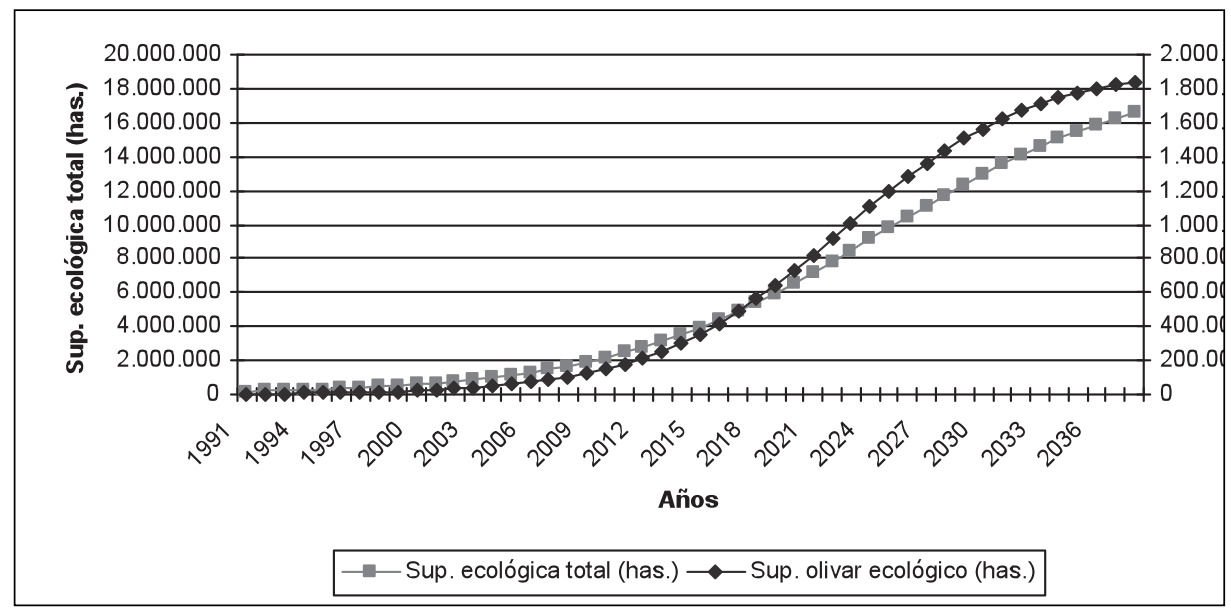

Nota: Se ha supuesto una proporción en torno al $10 \%$ de la superficie olivarera sobre la total durante el período que va desde 2009 a 2038. Estimaciones realizadas con SPSS v.14.

La Figura 3 muestra la senda de difusión logística simulada a partir de los supuestos que caracterizan el «escenario base». Según este modelo se estima que el punto de inflexión se alcanzará aproximadamente en el año 2017, momento en el que se contabilizarán más de 400 mil hectáreas de olivar ecológico. Los parámetros de los modelos simulados (logístico y Gompertz) aparecen en la Tabla 11.

TABLA 11.--Simulación de modelos de difusión de influencia interna para la superficie ecológica total y olivarera en España

\begin{tabular}{|c|c|c|c|c|}
\hline \multirow{2}{*}{ Parámetros } & \multicolumn{2}{|c|}{ Simulación logística: $\mathrm{M} /(1+\exp (\mathrm{a}-\mathrm{b} * \mathrm{t}))$} & \multicolumn{2}{c|}{ Simulación Gompertz: $\mathrm{M} * \exp (-\exp (\mathrm{a}-\mathrm{b} * \mathrm{t}))$} \\
\cline { 2 - 5 } & Has. totales & Has. olivar & Has. totales & Has. olivar \\
\hline $\mathrm{M}$ & 19.163 .079 & 1.923 .562 & 22.688 .915 & 2.238 .879 \\
\hline $\mathrm{a}$ & 5,684 & 6,412 & 2,747 & 3,141 \\
\hline $\mathrm{b}$ & 0,140 & 0,197 & 0,072 & 0,102 \\
\hline
\end{tabular}

La Tabla 12 resume los coeficientes de penetración simulados del modelo logístico. Anualmente por cada 15 hectáreas ecológicas una nueva hectárea se convierte al sistema ecológico. Mientras que son necesarias 400 hectáreas de olivar ecológico para incorporar cada año una nueva hectárea. 
TABLa 12.- Simulación de los coeficientes de penetración de la difusión logística de la superficie ecológica total y olivarera en España

\begin{tabular}{|l|l|l|}
\hline & Has totales & Has olivar \\
\hline por cada $1 \%$ & $0,15 \%$ & $0,21 \%$ \\
\hline por cada 1 hectárea & $0,0678 \%$ & $0,0025 \%$ \\
\hline $\mathbf{M}^{*}$ & 95,815 & 96,178 \\
\hline b & 0,140 & 0,197 \\
\hline
\end{tabular}

Nota: El valor de $\mathrm{M}^{*}$ se ha calculado como porcentaje de los valores máximos estimados de superficie en el escenario base, 20 millones de hectáreas ecológicas totales y 2 millones de olivar ecológico (80\% de la superficie convencional en ambos casos, suponiendo a partir de 2008 una banda de crecimiento estacionario entorno a los 25 millones de hectáreas totales de SAU y de 2,5 millones en olivar).

\section{CONCLUSiones}

De la revisión de la legislación agroambiental comunitaria y andaluza se observa el acertado tratamiento unificando las medidas de lucha contra la erosión y de reconversión de la explotación hacia el sistema productivo ecológico para el olivar en pendiente. No obstante se perciben potenciales deficiencias a partir del análisis empírico realizado a olivicultores de las provincias de Granada y Jaén en el año 2004.

Concretamente, del modelo probit estimado sobre los factores que explican la decisión del agricultor de reconvertir su explotación hacia el sistema ecológico destaca la influencia negativa de los rendimientos y la influencia positiva de una dedicación reciente y parcial a la actividad agraria. Curiosamente, la principal práctica de conservación adoptada en la zona, el no laboreo, no influye sobre el proceso de reconversión productiva. En cambio sí se incrementa la probabilidad con la adopción de la técnica de mantenimiento de pedrizas o muretes. La principal característica de esta técnica es su proceso de difusión logística, en consonancia con los resultados obtenidos para otras prácticas de conservación de suelos y en otras zonas de Andalucía por Parra y Calatrava-Requena (2005) y Franco (2009).

Del ejercicio de simulación se desprende que hay una gran diferencia entre las tasas de difusión a nivel de superficie de prácticas de conservación de suelos (como el mantenimiento de pedrizas) y la superficie olivarera ecológica, lo que por un lado es indicativo de la necesidad de dotar de mayor cuantía las ayudas de la medida agroambiental 
correspondiente (incluyendo no sólo las primas a los agricultores, sino también los servicios de asesoramiento); y por otro vincular más estrechamente la adopción conjunta de prácticas de conservación de suelos complementarias y la producción ecológica.

Para contrastar los resultados aquí obtenidos serían necesarios nuevos estudios que analicen la respuesta de los agricultores a los paquetes de medidas agroambientales tras la implantación de la nueva normativa sobre producción ecológica y control de la erosión recogida en los Planes de Desarrollo Rural, y particularmente el PDR (20072013) de Andalucía.

\section{REFERENCIAS BIBLIOGRÁFICAS}

Alonso, A. y Simón, X. (2008): «Evolución de la agricultura ecológica en Europa». Actas II Congreso de Agroecoloxía e Agricultura Ecolóxica en Galiza. Simón, X. y Capena, D. (coords). (2009). Construíndo un rural agroecolóxico. Universidade de Vigo. Vigo.

Alonso, A., Knickel, K. y Parrott, N. (2002): «Influencia de los canales comerciales en el desarrollo de la agricultura ecológica en Europa». V Congreso de la SEAE y I Congreso Iberoamericano de Agroecología. Gijón, 16-21 septiembre 2002, Tomo II, págs. 1409-1418.

Barea, F. y Ruíz, P. (2002): «Olivar ecológico en zonas de montaña andaluzas. Evolución y perspectivas». Actas del Forum Internacional BIOL 2002. Andria, Italia, abril 2002.

Calatrava-Requena, J. (2002): «Actitudes del consumidor español respecto a los productos ecológicos». Monografía. Consejería de Agricultura y Pesca. Junta de Andalucía. Sevilla.

Calatrava-Requena, J. y GonzÁlez, M. (2008): «Technical versus institutional innovation in Andalusian olive tree orchards». 12th Congress of European Association of Agricultural Economists. Ghent (Belgium), 26-29 August 2008.

Comisión Europea (2008): Campaña sobre la agricultura ecológica. Comisión Europea. Foire de Libramont, Bélgica. Disponible en www.organic-farming.europa.eu (Fecha de consulta: 23/10/2008).

David, P. A. (1969): «A contribution to the theory of diffusion». Standford Center for Research in Economic Growth. Memorandum 7. Standford, California.

Feder, G., Just, R. E. y Zilberman, D. (1985): «Adoption of agricultural innovations in developing countries. A survey». Economic Development and Cultural Change 33(2): 255-297.

Franco, J. A. (2009): Análisis económico de la erosion de suelos agrarios en el olivar del Alto Genil granadino. Tesis doctoral. Universidad de Córdoba.

Gil, J. M., Soler, F., Diéz, I., Sánchez, M., Sanjuán, A., Ben KaAbia, M. y Gracia, A. (2000): Potencial del mercado de los productos ecológicos en Aragón (II). Diputación General de Aragón. Zaragoza. 
Gould, B. W., SAupe, W. E. y Klemme, R. M. (1989): «Conservation tillage: the role of farm and operator characteristics and the perception of soil erosion». Land Economics 65: 167-182.

GrILICHES, Z. (1957): «Hybrid corn: an exploration in the economics of technological change». Econometrica 25: 501-522.

Hayami, Y. y Ruttan, V.W. (1971): Agricultural development: an international perspective. John Hopkins Press. Baltimore.

Hooks, G. M., NAPIER, T. L. y CARTER, M. V. (1983): «Correlates of adoption behaviors: the case of farm technologies». Rural Sociology 48(2): 308-323.

InNIS, H. (1927): The fur-trade of Canada. University of Toronto Library. Toronto.

MAPA (varios años): Estadísticas de agricultura ecológica. MAPA y Ministerio de Medio Ambiente y Medio Rural y Marino. Madrid.

Myint, H. (1958): «The Classical Theory of International Trade and the Underdeveloped Countries». Economic Journal 68: 317-337.

PARRA, C. (2003): Sistemas de producción ecológica, integrada y convencional en olivar: estudio de difusión de innovaciones y evaluación multifuncional. Tesis doctoral. Universidad de Córdoba.

Parra, C. y Calatrava-Requena, J. (2005): «Factors related to the adoption of organic farming in Spanish olive orchards». Spanish Journal of Agricultural Research 3(1): 5-16.

PAYSON, A. (1923): «Soil fertility, soil exhaustion and their historical significance». Quarterly Journal of Economics 37: 385-411.

Rogers, E. M. (1962): Diffusion of Innovations. The Free Press. New York.

Ryan, B. y Gross, N.C. (1943): «The diffusion of hybrid seed corn in two Iowa communities». Rural Sociology 8: 15-24.

Schultz, T. W. (1932): «Diminishing returns in view of progress in agricultural production». Journal of Farm Economics 14(October): 640-649.

- (1964): Transforming traditional agriculture. Yale University Press. New Haven, Connecticut.

Stoneman, P. (1981): «Intra firm diffusion, bayesian learning and profitability». Economic Journal 91: 375-388.

Von Thunen, J. H. (1966): Von Thunen's isolated state. Pergamon Press. Oxford. 